\title{
THE AUTOMORPHISM GROUP OF A GRAPH PRODUCT OF GROUPS
}

\author{
Martin R. Pettet \\ Department of Mathematics \\ University of Toledo \\ Toledo, Ohio 43606
}

\begin{abstract}
A theorem of Karrass, Pietrowski and Solitar on the structure of the automorphism group of an amalgamated free product is extended to automorphism groups of fundamental groups of graphs of groups in which the edge groups are incomparable up to conjugacy.
\end{abstract}

\section{Introduction}

The primary motivation for this note is the following theorem of Karrass, Pietrowski and Solitar [1]: Let $G$ be an amalgamated free product $H *_{U} K$, where $H \neq U \neq K$ and assume that $U$ is conjugate maximal in each of $H$ and $K$ (i.e. no conjugate of $U$ in either factor properly contains $U$ ). If $A$ is the group of automorphisms of $G$ which map each of the factors $H$ and $K$ to a conjugate of either itself or the other factor, then $A$ also admits an amalgamated free product decomposition.

It seems natural to ask to what extent an analogous conclusion holds for products involving more than two factors or, more generally, for fundamental groups of graphs of groups (the "graph products" of the title). In this paper an extension of the Karrass-Pietrowski-Solitar result is obtained by replacing conjugate maximality with a hypothesis on the edge groups (the "EGI hypothesis") which is generally more restrictive but which, in the case of two factors (and also HNN extensions), is equivalent to it. In essence, we show that if $G$ is the fundamental group of a graph of groups which satisfies this hypothesis and if $A$ is the group of those automorphisms of $G$ which map vertex groups to conjugates of vertex groups then the action of $G$ on the associated standard tree extends to an action of $A$ and hence, $A$ admits a combinatorial decomposition resembling that of $G$.

The argument depends heavily on basic results from the Bass-Serre theory of group actions on trees. Most of our notation and terminology follows that of $[2]$.

If $G$ is a group, an inverted edge of a $G$-graph is one which is mapped to itself by some element of $G$ but with the orientation reversed (i.e. with the incident vertices interchanged). Such an edge is subdivided by adding a new vertex at its mid-point and replacing it with two adjacent edges which are 
interchanged by the elements of $G$ which inverted the original edge. (Subdivision of the inverted edges is thus a topologically harmless device for eliminating inversions from a $G$-graph.)

We consider a graph of groups $(G(-), D)$ where $D$ is a connected directed graph and for $\mathbf{v} \in V D$ and $\mathbf{e} \in E D, G(\mathbf{v})$ and $G(\mathbf{e})$ denote the corresponding vertex and edge groups (which will be treated as subgroups of the fundamental group $\left.G=\pi_{1}(G(-), D)\right) . G(\mathbf{e})$ is taken to be a subgroup of $G(\mathbf{v})$ if $\mathbf{v}$ is the initial vertex of $\mathbf{e}$. The edge labels serve also as names of embeddings and as names of certain elements of the generating set of $G$. Thus, if $\mathbf{v}$ is the terminal vertex of e, then e denotes both an embedding of $G(\mathbf{e})$ in $G(\mathbf{v})$ and also an element of $G$ (possibly the identity) which conjugates elements of $G(\mathbf{e})$ to their images in $G(\mathbf{v})$ under the map e. Of course, in the case that $G$ is an amalgamated free product, $D$ is simply a segment and in the case of an HNN extension, it is a loop.

Some additional ad hoc terminology will facilitate the statement of the theorem. If $\mathbf{v}$ is a vertex of $D$, the subgroups of $G(\mathbf{v})$ of the form $G(\mathbf{e})$ (where $\mathbf{e}$ is an outgoing edge) and $G(\mathbf{e})^{\mathbf{e}}$ (where $\mathbf{e}$ is an incoming edge) will both be called $\mathbf{v}$-incident edge groups corresponding to e. We shall consider only graphs of groups which are proper in the sense that for each vertex $\mathbf{v}$ of $G$, every $\mathbf{v}$-incident edge group which does not correspond to a loop at $\mathbf{v}$ is a proper subgroup of $G(\mathbf{v})$. One class of proper graphs of groups which we will need to avoid is those in which $D$ consists of a single loop with both edge groups equal to the full vertex group. These we shall refer to as degenerate loops. (If $(G(-), D)$ is a degenerate loop then $G$ is simply a semidirect product of the vertex group $G(\mathbf{v})$ with an infinite cyclic group and $A=N_{A u t G}(G(\mathbf{v}))$.)

The central hypothesis of the paper is the following:

Definition. We shall say that $(G(-), D)$ satisfies the edge group incomparability (or EGI) hypothesis if, for each vertex $\mathbf{v}$ of $D$, no $\mathbf{v}$-incident edge group is conjugate in $G(\mathbf{v})$ to a subgroup of another $\mathbf{v}$-incident edge group unless the two are identical and correspond to the same edge of $D$.

For example, the EGI hypothesis is obviously satisfied if the edge groups are all finite with none having order divisible by the order of another.

If $\Gamma$ is any directed graph, we will denote the underlying undirected graph by $\Gamma_{0}$. Our main objective is

Theorem 1 Let $G$ be the fundamental group of a proper graph of groups $(G(-), D)$ which satisfies the EGI hypothesis and is not a degenerate loop. Let $T$ be the corresponding standard $G$-tree and let $A$ be the group of all automorphisms of $G$ which map each vertex group of $(G(-), D)$ to a $G$ conjugate of some vertex group. Then $T$ is an InnG-tree and the action of Inn $G$ on the underlying undirected tree $T_{0}$ extends to an action of $A$ which, in turn, induces an action of $A / \operatorname{Inn} G$ on the undirected graph $D_{0}$. 
As a consequence, $A$ is isomorphic to the fundamental group of a proper graph of groups $\left(A(-), D^{*}\right)$ which satisfies the EGI hypothesis and such that the following hold:

(i) $D_{0}^{*}$ is isomorphic to the quotient modulo $A$ of the A-graph obtained from $D_{0}$ by subdividing all inverted edges.

(ii) If $\overline{\mathbf{v}} \in V D_{0}^{*}$ is the image of a vertex $\mathbf{v}$ of $D_{0}$ then $A(\overline{\mathbf{v}})=N_{A}(G(\mathbf{v}))$. If $\overline{\mathbf{v}} \in V D_{0}^{*}$ arises from subdivision of an edge of $D_{0}$ which is inverted under the action of $A$ then $A(\overline{\mathbf{v}})$ consists of those elements of $A$ which either fix or invert this edge.

(iii) $\left|E D^{*}\right| \leq|E D|$. Moreover, if $D$ is a finite tree then so is $D^{*}$.

The inspiration for the proof of the theorem is the observation that if $(G(-), D)$ is a proper graph of groups which satisfies the EGI hypothesis and is not a degenerate loop, then there is a $G$-bijection between the vertex set $V T$ of the standard $G$-tree $T$ and the $A$-set of all vertex stabilizers in $G$ (Lemma 4.1). The bulk of the argument is concerned with establishing that under the EGI hypothesis, the action of $A$ on $V T$ induced by this bijection is actually isometric on the underlying undirected tree $T_{0}$ (Lemma 3.1).

As a simple application, let $H$ and $K$ be groups with proper non-trivial subgroups $U$ and $V$ respectively and consider the group

$$
G=\langle H, K:[u, v]=1 \forall u \in U, v \in V\rangle .
$$

If $L=U \times V$, then $G=H *_{U} L *_{V} K$ and since $U$ and $V$ are normal in $L$, the EGI hypothesis holds. Here the graph $D_{0}$ is

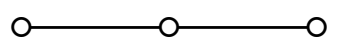

If the non-identity automorphism of $D_{0}$ is realized by an element of $A$ (the case if, for example, there is an isomorphism $\phi: H \rightarrow K$ such that $U^{\phi}=V$ ) then $D_{0}^{*}$ is a segment and Theorem 1 yields that $A$ is an amalgamated free product $N_{A}(H) *_{B} N_{A}(L)$, where $B=N_{A}(H) \cap N_{A}(L)$. Otherwise, $D_{0}^{*} \cong D_{0}$ and so $A$ has the form $N_{A}(H) *_{B} N_{A}(L) *_{C} N_{A}(K)$.

Theorem 1 is probably of greatest interest in the case that the subgroup $A$ is the full automorphism group of $G$. One additional hypothesis which ensures that $A=A u t G$ is that each vertex group of $(G(-), D)$ possess intrinsically the property that it fixes a vertex of any tree on which it acts. (This is termed property (FA) in [4] where it is shown to be characterized by three conditions: that $G$ have no non-trivial amalgamated free product decomposition, that it have no infinite cyclic quotient, and that it not be a union of a strictly ascending chain of proper subgroups.) In this case, if $v$ is a vertex of the $G$-tree $T$ and if $\alpha \in A u t G$, then $\left(G_{v}\right)^{\alpha} \subseteq G_{u}$ for 
some $u \in V T$ and similarly, $\left(G_{u}\right)^{\alpha^{-1}} \subseteq G_{w}$ for some $w \in V T$, whence $G_{v} \subseteq G_{w}$. The EGI hypothesis and the fact that $(G(-), D)$ is proper then force $v=w$ (Lemma 4.1) and so $\left(G_{v}\right)^{\alpha}=G_{u}$, proving that $\alpha$ maps vertex stabilizers to vertex stabilizers and so belongs to $A$. It follows, for example, that $A=A u t G$ if each vertex group of $(G(-), D)$ is finitely generated and periodic. (See 6.3.1 of [4].) Theorem 2.3 of [5] provides another criterion of this type which applies when the vertex groups are polycyclic-by-finite.

Following the completion of the proof of Theorem 1 in Section 4, we derive an analog of the Karrass-Pietrowski-Solitar theorem for proper HNN extensions $G=H *_{U} t$ (Theorem 2). This theme is pursued further in Section 5 where, in the special case that $U$ is centralized by $t$, a short computation leads to a very precise description of $A$ in terms of $H$ and $U$. The final observation in Section 4 is a natural extension of a result of E. Raptis, D. Varsos and O. Talelli on automorphism groups of amalgamated free products and HNN extensions of polycyclic-by-finite groups (Theorem 3).

\section{Preliminaries}

Let $G$ be a group acting on a set $X$. We shall say that the action is locally transitive if for every element $x$ of $X, N_{G}\left(G_{x}\right)$ is transitive on the set Fix $\left(G_{x}\right)$ of points fixed by the stabilizer $G_{x}$ of $x$ or, equivalently, if for any two elements $x$ and $y$ of $X, G_{x}$ is contained in $G_{y}$ only if $G_{x}=G_{y}$ and the orbits $x^{G}$ and $y^{G}$ are identical. In the context of finite permutation groups, this property is a familiar (and trivial) consequence of transitivity (although in general, local transitivity does not follow from transitivity without the additional hypothesis that each point stabilizer be conjugate maximal).

From the Bass-Serre theory, the group $G$ of Theorem 1 acts on the standard tree (or universal cover) $T$. Accordingly, most of the steps in the argument will be formulated in terms of group actions on trees. We say that the action of $G$ on a graph $\Gamma$ is locally edge-transitive if it is locally transitive on the edge set $E \Gamma$. If $e$ and $f$ are edges of the standard tree $T$ with $G_{e} \subseteq G_{f}$ and if $e=e_{1}, e_{2}, \ldots, e_{n}=f$ is the edge sequence in the geodesic connecting $e$ and $f$, then $G_{e}=G_{e} \cap G_{f} \subseteq G_{e_{i}}$ for $1 \leq i \leq n$. From this observation, the EGI hypothesis defined in the Introduction is easily seen to be simply a reformulation in terms of the graph of groups of the assumption that $G$ acts locally edge-transitively on $T$.

The following observation is crucial here: If $e$ is an edge of the $G$-tree $T$ and if $p$ and $q$ are any two vertices of $F_{i x}\left(G_{e}\right)$ then $G_{e}$ fixes each vertex and edge in the unique reduced path (or geodesic) between $p$ and $q$. (By $G_{e}$, we shall always mean the point-wise stabilizer of the edge $e$ viewed as a 1-simplex.) In particular, $G_{e}$ fixes each of the vertices incident with $e$.) It follows that $\mathrm{Fix}_{T}\left(G_{e}\right)$ is a $N_{G}\left(G_{e}\right)$-tree and so (see, for example, $\S 4$ and $\S 5$ of [4]) if $G$ acts locally edge-transitively and without inversion on $T$, then for each edge $e$ of $T$ with incident vertices $u$ and $v, N_{G}\left(G_{e}\right)$ is either an 
amalgamated free product $N_{G_{u}}\left(G_{e}\right) *_{G_{e}} N_{G_{v}}\left(G_{e}\right)$ (if $u$ and $v$ are not $N_{G}\left(G_{e}\right)$ conjugate) or an HNN-extension $N_{G_{v}}\left(G_{e}\right) *_{G_{e}} t$ (if $u^{t}=v$ for some $t \in$ $N_{G}\left(G_{e}\right)$ ). In particular, $N_{G}\left(G_{e}\right)$ is generated by those of its elements $g$ for which $e$ and $e^{g}$ are adjacent.

Let $T$ be a tree and let $d$ be the edge metric on $V T$ (so if $u$ and $v$ are vertices of $T, d(u, v)$ is the number of edges in the geodesic $[u, v]$ from $u$ to $v)$.

Lemma 2.1 If $G$ is a group and $T$ is a simplicial $G$-tree, then any element of $G$ which inverts an edge e neither fixes a vertex nor inverts any edge distinct from e.

Proof. This is an easy consequence of the $d$-isometric action of $G$ on $T$.

The thrust of the next lemma is that if $G$ is a group of automorphisms of a tree $T$, then (even without the hypothesis of local edge-transitivity) elements of $N_{S y m(V T)}(G)$ map edge stabilizers to edge stabilizers.

Lemma 2.2 Let $T$ be a simplicial tree, $G \subseteq$ AutT and $\alpha \in N_{\operatorname{Sym}(V T)}(G)$. Suppose that $e \in E T$ with incident vertices $p$ and $q$, and let $p^{\alpha}=u$ and $q^{\alpha}=v$. Then $\left(G_{e}\right)^{\alpha}=G_{f}$ for some edge $f$ in the geodesic $[u, v]$. Moreover, if $g \in G$ such that $g$ inverts the edge $e$, then $f$ may be chosen so that $g^{\alpha}$ inverts $f$.

Proof. We have

$$
\left(G_{e}\right)^{\alpha}=\left(G_{p} \cap G_{q}\right)^{\alpha}=G_{u} \cap G_{v} \subseteq G_{b}
$$

for every edge $b$ in the geodesic $[u, v]$. Since $p$ and $q$ lie in different connected components of $T \backslash\{e\}$, there is an edge $f$ in $[u, v]$ with incident vertices $x$ and $y$, say, such that $\alpha^{-1}$ maps $x$ and $y$ respectively to vertices $z$ and $w$ in different components of $T \backslash\{e\}$. Then $e$ lies in the geodesic $[z, w]$ and so $G_{z} \cap G_{w} \subseteq G_{e}$. It follows that

$$
G_{f}=G_{x} \cap G_{y}=\left(G_{z} \cap G_{w}\right)^{\alpha} \subseteq\left(G_{e}\right)^{\alpha},
$$

whence $\left(G_{e}\right)^{\alpha}=G_{f}$.

If $g$ inverts the edge $e\left(\right.$ so $p^{g}=q$ and $\left.q^{g}=p\right)$, then

$$
u^{g^{\alpha}}=p^{\alpha g^{\alpha}}=\left(p^{g}\right)^{\alpha}=q^{\alpha}=v .
$$

By a similar argument, $v^{g^{\alpha}}=u$. By Lemma 2.1, $g$ does not stabilize any vertex of $T$ and so neither does $g^{\alpha}$. Since $g^{\alpha}$ interchanges $u$ and $v$, it follows that it must invert some edge $f$ in $[u, v]$. Let $x$ and $y$ be the vertices incident with $f$ (so $g^{\alpha}$ interchanges $x$ and $y$ ) and let $x^{\alpha^{-1}}=z$ and $y^{\alpha^{-1}}=w$. Then

$$
z^{g}=x^{\alpha^{-1} g}=x^{g^{\alpha} \alpha^{-1}}=y^{\alpha^{-1}}=w .
$$


Similarly, $w^{g}=z$. Since $g$ fixes no vertex of $T$, it must invert an edge in the geodesic $[z, w]$ and so by Lemma $2.1, e$ is an edge in $[z, w]$. Thus, $z$ and $w$ lie in different components of $T \backslash\{e\}$ and, as in the previous paragraph, $\left(G_{e}\right)^{\alpha}=G_{f}$.

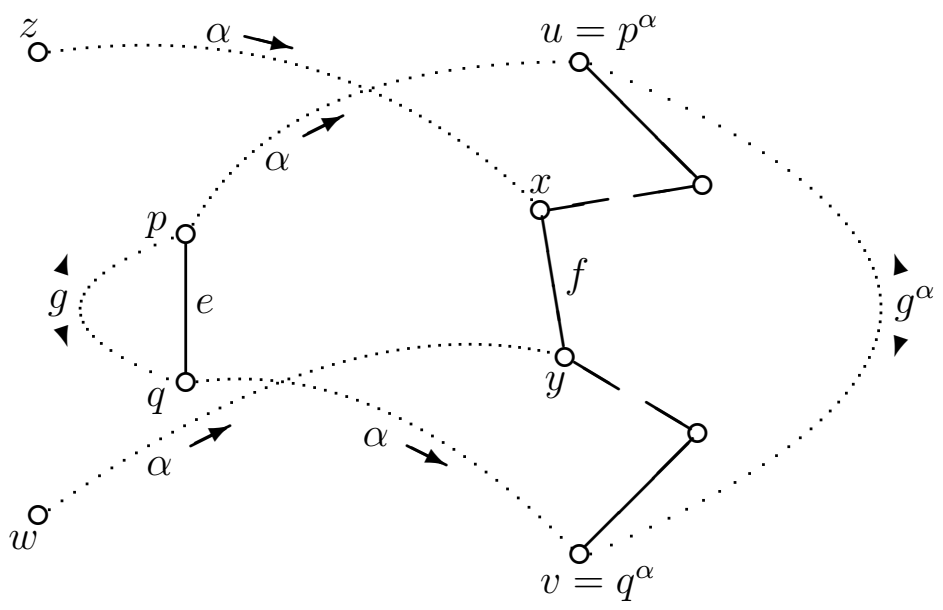

Lemma 2.3 Let $G$ be a group and $T$ be a $G$-tree. Let $\left\{u_{1}, u_{2}, \ldots u_{n}\right\}$ be a set of vertices of $T$ and let $p$ also be a vertex of $T$. If $g \in\left\langle G_{u_{1}}, G_{u_{2}}, \ldots G_{u_{n}}\right\rangle$, then $d\left(p, p^{g}\right) \equiv 0(\bmod 2)$.

Proof. First, observe that if $x, y, z$ are any three vertices of $T$, then

$$
d(x, y)+d(y, z) \equiv d(x, z)(\bmod 2) .
$$

We induct on the minimum length $m$ of an expression of $g$ as a product $g_{1} g_{2} \ldots g_{m}$ with consecutive terms belonging to distinct $G_{u_{j}}$ 's. If $m=1$, then $g \in G_{u_{i}}$ for some $i$ and so

$$
d\left(p, p^{g}\right) \equiv d\left(p, u_{i}\right)+d\left(u_{i}, p^{g}\right) \equiv 0(\bmod 2)
$$

because $d\left(u_{i}, p^{g}\right)=d\left(\left(u_{i}\right)^{g}, p^{g}\right)=d\left(u_{i}, p\right)$. If $m>1$, write $g=h a$, where $a \in G_{u_{i}}$ for some $i$ and $h$ has minimum length $m-1$. If $q=p^{h}$, then $d(p, q) \equiv 0(\bmod 2)$ by the inductive hypothesis and so

$$
d\left(p, p^{g}\right) \equiv d(p, q)+d\left(q, p^{g}\right) \equiv d\left(q, q^{a}\right) \equiv 0(\bmod 2) .
$$

Lemma 2.4 Let $T$ be a simplicial $G$-tree with no inversions. If $p$ and $q$ are adjacent vertices of $T$ sharing a common edge $e$, then $\left\langle G_{p}, G_{q}\right\rangle$ is an amalgamated free product $G_{p} *_{G_{e}} G_{q}$. If, in addition, $p^{g}=q$ for some $g \in G$, then $\left\langle G_{p}, g\right\rangle=\left\langle G_{q}, g\right\rangle$ is an $H N N$ extension $G_{q} *_{G_{e}} g$.

Proof. The set of all edges of $T$ which are conjugate to $e$ by an element of $\left\langle G_{p}, G_{q}\right\rangle$ is the edge set of a $\left\langle G_{p}, G_{q}\right\rangle$-subtree of $T$. Since $\left\langle G_{p}, G_{q}\right\rangle$ is edge transitive but (by Lemma 2.3) not vertex transitive on this subtree, the first conclusion follows from the Bass-Serre theory. If $p^{g}=q$, then the set of all 
edges of $T$ which are conjugate to $e$ by an element of $\left\langle G_{p}, g\right\rangle$ is a tree on which $\left\langle G_{q}, g\right\rangle$ is both edge and vertex transitive and again, the Bass-Serre theory yields the desired conclusion.

The next lemma (and its proof) is a slight variation on the key first step in [1].

Lemma 2.5 Let $G=H *_{U} K$. If $g \in N_{G}(U)$ such that $\left\langle H, K^{g}\right\rangle=G$ and $H \cap K^{g}=U$, then $K^{g}=K^{h}$ for some $h \in N_{H}(U)$.

Proof. This is trivial if $H=U$ or $K=U$. If $H \neq U \neq K$, let $X$ and $Y$ be right transversals for $U$ in $H$ and $K$ respectively and assume that each contains 1. It is sufficient to prove that $g=k h$ for some $h \in H$ and $k \in K$ (for if so, $U^{h}=(H \cap K)^{h}=H \cap K^{h}=H \cap K^{g}=U$, whence $\left.h \in N_{H}(U)\right)$. If this is not so, then without loss of generality we may assume that for some $n \geq 1$, $g=x_{1} y_{1} x_{2} y_{2} \ldots x_{n} y_{n}$ where $x_{i} \in X \backslash\{1\}$ and $y_{i} \in Y \backslash\{1\}$ for $1 \leq i \leq n$. But if $h_{j} \in X \backslash\{1\}$ and $k_{j} \in Y \backslash\{1\}$ for $1 \leq j \leq m$, then $h_{1} k_{1}^{g} h_{2} k_{2}^{g} \ldots h_{m} k_{m}^{g}$ is reduced and in particular, no such element belongs to $K$. It follows that $\left\langle H, K^{g}\right\rangle \cap K=U$ which contradicts the assumption that $\left\langle H, K^{g}\right\rangle=G$.

Finally in this section, we prove a result analogous to Lemma 2.5 for HNN extensions. Similar results (and proofs) appear in [3] and in [5].

Lemma 2.6 Let $G$ be an $H N N$ extension $H *_{U} t$, where $t$ normalizes the subgroup $U$. If $G=\langle H, s\rangle$ for some $s \in N_{G}(U)$, then $s=a t^{\varepsilon} b$ for some $a, b \in H$ and $\varepsilon \in\{-1,1\}$.

Proof. Let $X$ be a right transversal for $U$ in $H$ which contains the identity, whence $s$ has a unique $t$-reduced representation $h t^{\varepsilon_{1}} x_{1} \ldots t^{\varepsilon_{n}} x_{n}$ where $h \in H$ and where for $1 \leq i \leq n$, the $x_{i}$ 's are elements of $X$ and the $\varepsilon_{i}$ 's are \pm 1 . The claim of the lemma is that $n=1$ so assume otherwise, that $n>1$. Clearly, it is no loss to assume that $h=x_{n}=1$ and so $s=t^{\varepsilon_{1}} h_{1} \ldots t^{\varepsilon_{n}}$. If $N$ is the normal closure of $H$ in $G$, then $G / N$ is infinite cyclic with both $t N$ and $s N$ being generators and so $s \equiv t^{ \pm 1}(\bmod N)$. Thus, $\sum_{i=1}^{n} \varepsilon_{i}= \pm 1$ and in particular, $n$ is odd.

Computing a $t$-reduced expression for a positive power of $s$ (or any element of $G$ ) involves eliminating $t$ 's and $t^{-1}$ 's in pairs and hence, at most $(n-1) / 2 t$-reductions can occur between consecutive occurrences of the expression for $s$ in such a power. In other words, the power of $t$ in the exact middle of each occurrence of the expression for $s$ cannot be absorbed into any $t$-reductions. Since $n>1$, it follows that the $t$-reduced expression for any non-trivial power of $s$ has the form $u t^{\varepsilon_{1}} x_{1} w x_{n-1} t^{\varepsilon_{n}}$, where $u \in U$ and $w$ involves at least one non-trivial power of $t$.

Because $G=\langle H, s\rangle$ and $s \in N_{G}(U)$, we may write $t=g s^{e_{1}} y_{1} \ldots s^{e_{m}} y_{m}$ where $g \in H$, the $y_{i}$ 's are non-identity elements of $X$ and the $e_{i}$ 's are nonzero integers for $1 \leq i \leq m$. From the conclusion obtained in the preceding 
paragraph about the reduced form of each non-trivial power of $s$, it follows that non-trivial powers of $t$ must occur at least $3 m$ times in the $t$-reduced form of $g s^{e_{1}} y_{1} \ldots s^{e_{m}} y_{m}$. This obviously contradicts the fact that this expression represents $t$ itself.

\section{Locally edge-transitive actions}

A concisely formulated technical lemma represents the crux of the argument.

Lemma 3.1 Let $T$ be an undirected simplicial tree and suppose that $G$ is a locally edge-transitive subgroup of AutT. Then $N_{S y m(V T)}(G) \subseteq A u t T$.

Proof. First we argue that it is sufficient to restrict our attention to the case that the action of $G$ on $T$ involves no edge inversions.

Let $T^{*}$ be the $G$-tree obtained from $T$ by subdividing each inverted edge (and only these edges). The action of $G$ on $T^{*}$ is still faithful and locally edge-transitive (since the set of edge-stabilizers is not changed) and there are, of course, no edge inversions. Let $A=N_{S y m(V T)}(G)$. With $G$ identified as a subgroup of $A u t T^{*}$, we must now show that $A$ can be identified with a subgroup of $N_{S y m\left(V T^{*}\right)}(G)$.

If $w$ is the mid-point of an edge $e \in E T$ such that $e$ is inverted by $g \in G$ (so $g \in N_{G}\left(G_{e}\right)$ ), then $G_{w}=G_{e}\langle g\rangle$. If $\alpha \in A$, Lemma 2.2 implies that there is an edge $f$ such that $\left(G_{e}\right)^{\alpha}=G_{f}$ and $g^{\alpha}$ inverts $f$, whence

$$
\left(G_{w}\right)^{\alpha}=\left(G_{e}\right)^{\alpha}\left\langle g^{\alpha}\right\rangle=G_{f}\left\langle g^{\alpha}\right\rangle=G_{z}
$$

where $z$ is the midpoint of $f$. This vertex $z$ of $T^{*}$ is uniquely determined by $w$ and $\alpha$, for if $G_{z}=G_{x}$ with $x \in V T^{*}$, then $g^{\alpha}$ fixes $x$ but fixes no vertex of $T$ (Lemma 2.1), whence $x$ is the mid-point of an edge $b$ of $T$. Then $g^{\alpha}$ must either invert or fix $b$, which is only possible if $b=f$ and $x=z$ (again by Lemma 2.1). Thus, if $w$ is the mid-point of an edge which is inverted by $g \in G$, we may define $w^{\alpha}$ to be the mid-point of the unique edge of $T$ which is inverted by $g^{\alpha}$. It is routine to check that this defines an action of $A$ on the set of mid-points of inverted edges of $T$ and so $A$ may be identified as a subgroup of $N_{\text {Sym }\left(V T^{*}\right)}(G)$.

The upshot of this is that if we can show that $N_{\operatorname{Sym}\left(V T^{*}\right)}(G) \subseteq A u t T^{*}$, then $A \subseteq A u t T^{*}$ and, because $V T$ is $A$-invariant, it will follow that $A \subseteq A u t T$ as required. Therefore, we may assume for the remainder of the proof that $T=T^{*}$ (that is, that $G$ acts without inversion on $T$ ).

We must show that for any $\alpha \in A$ and any pair $p, q$ of adjacent vertices of $T$, the images $p^{\alpha}$ and $q^{\alpha}$ are also adjacent. Suppose that $p$ and $q$ are such a pair of vertices sharing a common edge $e$. Let $u=p^{\alpha}$ and $v=q^{\alpha}$ and let $f$ be the first edge in the geodesic $[u, v]$ and $w$ be the first vertex out of $u$ in this geodesic (so $f$ is incident with $u$ and $w$ ). Then $\left(G_{e}\right)^{\alpha}=\left(G_{p} \cap G_{q}\right)^{\alpha}=G_{u} \cap G_{v}$ is an edge stabilizer (Lemma 2.2) which fixes every edge in $[u, v]$. Therefore, 
local edge-transitivity yields that all edges in $[u, v]$ are conjugate to $f$ under $N_{G}\left(G_{f}\right)$ (and hence, have stabilizer $G_{f}$ ). In particular, $\left(G_{e}\right)^{\alpha}=G_{u} \cap G_{v}=$ $G_{f}$. We now consider two cases:

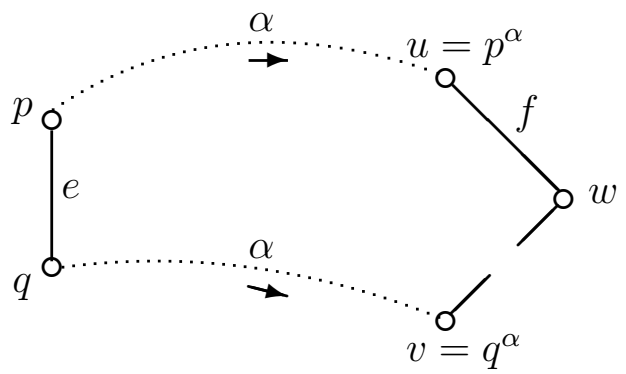

Case 1. $p$ and $q$ are not $N_{G}\left(G_{e}\right)$-conjugate.

In this case, $u$ is certainly not $N_{G}\left(G_{f}\right)$-conjugate to $v$ and so, since all edges in $[u, v]$ are $N_{G}\left(G_{f}\right)$-conjugate, $w$ must be $N_{G}\left(G_{f}\right)$-conjugate to $v$ (and not to $u$ ). Let $g$ be an element of $N_{G}\left(G_{f}\right)$ such that $w^{g}=v$. Since $u$ and $w$ are adjacent but not conjugate in $N_{G}\left(G_{f}\right)$, local edge-transitivity implies that $N_{G}\left(G_{f}\right)=N_{G_{u}}\left(G_{f}\right) *_{G_{f}} N_{G_{w}}\left(G_{f}\right)$ and in particular, $g \in N_{G}\left(G_{f}\right) \subseteq\left\langle G_{u}, G_{w}\right\rangle$. Thus,

$$
\left\langle G_{u}, G_{v}\right\rangle=\left\langle G_{u},\left(G_{w}\right)^{g}\right\rangle \subseteq\left\langle G_{u}, G_{w}\right\rangle .
$$

On the other hand, $g^{-1}=x^{\alpha}$ for some $x$ in $N_{G}\left(G_{e}\right)$. Again by local edge-transitivity, $N_{G}\left(G_{e}\right) \subseteq\left\langle G_{p}, G_{q}\right\rangle$ and in particular, $x \in\left\langle G_{p}, G_{q}\right\rangle$. Thus,

$$
\left\langle G_{u}, G_{w}\right\rangle=\left\langle G_{p},\left(G_{q}\right)^{x}\right\rangle^{\alpha} \subseteq\left\langle G_{p}, G_{q}\right\rangle^{\alpha}=\left\langle G_{u}, G_{v}\right\rangle
$$

It follows from Lemma 2.4 that

$$
G_{u} *_{G_{f}} G_{w}=\left\langle G_{u}, G_{w}\right\rangle=\left\langle G_{u}, G_{v}\right\rangle=\left\langle G_{u},\left(G_{w}\right)^{g}\right\rangle .
$$

Because

$$
G_{f}=G_{u} \cap G_{v}=G_{u} \cap\left(G_{w}\right)^{g},
$$

Lemma 2.5 yields that $G_{v}=\left(G_{w}\right)^{g}=\left(G_{w}\right)^{h}$ for some $h \in N_{G_{u}}\left(G_{f}\right)$. Since $w^{h}$ is adjacent to $u^{h}=u$, the geodesic $[w, v]$ is contained in $\left[w^{h}, v\right]$. Because each edge in $[u, v]$ (and hence, in $[w, v]$ ) has stabilizer $G_{f}$, we conclude that if $v \neq w\left(\right.$ or $\left.w^{h} \neq w\right)$, then

$$
G_{v}=\left(G_{w}\right)^{h} \cap G_{v} \subseteq G_{f} \subseteq G_{u} .
$$

But if $G_{v} \subseteq G_{u}$, then

$$
g \in\left\langle G_{u}, G_{w}\right\rangle=\left\langle G_{u}, G_{v}\right\rangle=G_{u}
$$

whence, $d(u, v)=d\left(u^{g}, w^{g}\right)=d(u, w)=1$ and so $v=w$. Thus, we are forced to the conclusion that $v=w$ in all cases, whence $u$ and $v$ are adjacent in $T$.

Case 2. $p$ and $q$ are $N_{G}\left(G_{e}\right)$-conjugate. 
Let $p=q^{t}$ where $t \in N_{G}\left(G_{e}\right)$. Then $N_{G}\left(G_{e}\right)=N_{G_{p}}\left(G_{e}\right) *_{G_{e}} t$ and so $N_{G}\left(G_{f}\right)=N_{G}\left(G_{e}\right)^{\alpha}=N_{G_{u}}\left(G_{f}\right) *_{G_{f}} t^{\alpha}$. Also,

$$
u^{t^{\alpha}}=\left(p^{\alpha}\right)^{t^{\alpha}}=\left(p^{t}\right)^{\alpha}=q^{\alpha}=v .
$$

We claim first that $u$ and $w$ are $N_{G}\left(G_{f}\right)$-conjugate. For if not, then the action of $N_{G}\left(G_{f}\right)$ on $\operatorname{Fix}\left(G_{f}\right)$ is not vertex-transitive and so

$$
t^{\alpha} \in N_{G}\left(G_{f}\right)=N_{G_{u}}\left(G_{f}\right) *_{G_{f}} N_{G_{w}}\left(G_{f}\right) \subseteq\left\langle G_{u}, G_{w}\right\rangle .
$$

Applying $\alpha^{-1}$, we conclude that $t \in\left\langle G_{p}, G_{r}\right\rangle$, where $r^{\alpha}=w$, which contradicts Lemma 2.3 since $d\left(p, p^{t}\right)=d(p, q)=1$.

Let $s \in N_{G}\left(G_{f}\right)$ such that $u=w^{s}$ (and so by Lemma 2.4, $N_{G}\left(G_{f}\right)$ is an HNN extension $\left.N_{G_{u}}\left(G_{f}\right) *_{G_{f}} s\right)$. Then $t^{\alpha} \in N_{G}\left(G_{f}\right)=\left\langle N_{G_{u}}\left(G_{f}\right), s\right\rangle$ and so $\left\langle G_{u}, t^{\alpha}\right\rangle \subseteq\left\langle G_{u}, s\right\rangle$. But

$$
s^{\alpha^{-1}} \in N_{G}\left(G_{f}\right)^{\alpha^{-1}}=N_{G}\left(G_{e}\right)=N_{G_{p}}\left(G_{e}\right) *_{G_{e}} t \subseteq\left\langle G_{p}, t\right\rangle .
$$

Therefore,

$$
s \in\left\langle G_{p}, t\right\rangle^{\alpha}=\left\langle G_{u}, t^{\alpha}\right\rangle
$$

and so $\left\langle G_{u}, s\right\rangle \subseteq\left\langle G_{u}, t^{\alpha}\right\rangle$. Hence, $\left\langle G_{u}, s\right\rangle=\left\langle G_{u}, t^{\alpha}\right\rangle$.

Since, by Lemma 2.4, $\left\langle G_{u}, s\right\rangle=G_{u} *_{G_{f}} s$, it follows from Lemma 2.6 that $t^{\alpha}=a s^{\varepsilon} b$, where $a, b \in G_{u}$ and $\varepsilon \in\{1,-1\}$. Therefore,

$$
d(u, v)=d\left(u, u^{t^{\alpha}}\right)=d\left(u, u^{a s^{\varepsilon} b}\right)=d\left(u, u^{s^{\varepsilon}}\right) .
$$

If $\varepsilon=-1$, then

$$
d(u, v)=d\left(u, u^{s^{-1}}\right)=d(u, w)=1
$$

and if $\varepsilon=1$, then

$$
d(u, v)=d\left(u, u^{s}\right)=d\left(u^{s^{-1}}, u\right)=d(w, u)=1,
$$

so in either case, $u$ and $v$ are adjacent. This completes the proof that $\alpha$ is actually a $d$-isometry of the (unoriented) tree $T$ and hence, that $A \subseteq A u t T$. Of course, in considering the implications of this conclusion for the structure of $A$, we must allow for the possibility that the action of $A$ involves edge inversions, even if that of $G$ is inversion-free.

The following observation about local transitivity is stated in somewhat greater generality than is strictly necessary for the proof of Theorem 1 but the price is only a short induction argument.

Lemma 3.2 Let $A \subseteq \operatorname{Sym}(X)$ for some set $X$. If $A$ contains a locally transitive ascendant subgroup $G$, then $A$ itself is locally transitive. 
Proof. Let $G=G_{1} \unlhd G_{2} \unlhd \ldots \unlhd G_{\beta}=A$ be an ascending series. If the lemma is false, let $\delta$ be the smallest ordinal for which $G_{\delta}$ is not locally transitive. Let $K=G_{\delta}$.

Suppose $\delta$ is a limit ordinal. If $x, y \in X$ with $K_{x} \subseteq K_{y}$, then for every $\gamma<\delta, G_{\gamma}$ is locally transitive and so $\left(G_{\gamma}\right)_{x}=\left(G_{\gamma}\right)_{y}$. Since $K$ is the union of all such $G_{\gamma}$ 's, it follows that $K_{x}=K_{y}$, and this contradicts the assumption that $K$ is not locally transitive.

Assume now that $\delta$ is not a limit ordinal and let $H=G_{\delta-1}$. If $x, y \in X$ with $K_{x} \subseteq K_{y}$, then $H_{x} \subseteq H_{y}$ and hence, by the local transitivity of $H$, $H_{x}=H_{y}$ and $y=x^{g}$ for some $g \in N_{H}\left(H_{x}\right)$ (so $\left.x^{K}=y^{K}\right)$. But $K_{y} \subseteq K_{x} H$, for if $\alpha \in K_{y}=\left(K_{x}\right)^{g}$ then

$$
\alpha=\beta^{g}=\beta[g, \beta]^{-1} \in K_{x} H
$$

for some $\beta \in K_{x}$. Therefore,

$$
K_{y}=K_{x} H \cap K_{y}=K_{x}\left(H \cap K_{y}\right)=K_{x} .
$$

This again contradicts the assumption that $K=G_{\delta}$ is not locally transitive.

Lemma 3.2 suggests the following slight refinement of Lemma 3.1 which follows from it by transfinite induction:

Corollary 3.3 Let $T$ be a simplicial tree and suppose that $G$ is a locally edgetransitive subgroup of AutT. If $\alpha \in \operatorname{Sym}(V T)$ such that $G$ is an ascendant subgroup of $\langle G, \alpha\rangle$, then $\alpha \in$ AutT.

\section{The main results}

Lemma 4.1 Let $(G(-), D)$ be a proper graph of groups which satisfies the EGI hypothesis and is not a degenerate loop. Let $T$ be the corresponding standard $G$-tree. If $u, v \in V T$ such that $G_{u} \subseteq G_{v}$ then $u=v$.

Proof. Let $e$ be the first edge out of $u$ in the geodesic from $u$ to $v$ and let $w$ be the first vertex. If $f$ is any edge incident with $u$ then

$$
G_{f} \subseteq G_{u}=G_{u} \cap G_{v} \subseteq G_{e} \subseteq G_{w}
$$

By the EGI hypothesis, $f$ is $G$-conjugate to $e$ and $G_{f}=G_{e}=G_{u}$. If $\mathbf{u}$ and e are the projections of $u$ and $e$ in $D$, then because $(G(-), D)$ is proper, e is a loop at $\mathbf{u}$ and $\mathbf{e}$ is the only edge of $D$ which is incident with $\mathbf{u}$. Because $D$ is connected, e must be the only edge in $D$ and $(G(-), D)$ is a degenerate loop, a contradiction.

Proof of Theorem 1. Let $(G(-), D), G, T$ and $A$ satisfy the hypotheses of Theorem 1. By Lemma 4.1, the $G$-map $v \mapsto G_{v}$ is a bijection between the 
vertex set $V T$ and the set of vertex stabilizers in $G$. In particular, elements of the center of $G$ must act trivially on $V T$. Thus, $T_{0}$ is an InnG-tree (with every inner automorphism $i_{g}$ having the same action as $g \in G$ ) and the bijection induces an action of $A$ on $V T$ which extends the action of $\operatorname{Inn} G$. Of course, the EGI hypothesis is equivalent to the assumption that $G$ (and hence, Inn $G$ ) acts locally edge-transitively on $T$.

Thus, Lemma 3.1 implies that $A$ induces a group of automorphisms of the undirected tree $T_{0}$ and so, by the Bass-Serre theory, $A$ also admits a presentation as the fundamental group of a certain graph of groups $\left(A(-), D^{*}\right)$. The graph $D^{*}$ in this presentation may be taken to be the quotient $T^{*} / A$, where $T^{*}$ is the directed $A$-tree obtained from $T$ by subdividing each inverted edge of $T$ and orienting both newly created edges as outgoing from the new vertex.

Indeed, $A$ induces a group of automorphisms of the undirected quotient graph $D_{0}=T_{0} / G$ and, because $A$ contains the group Inn $G$ of inner automorphisms of $G$, an edge of $T_{0}$ is inverted by an element of $A$ if and only if the projection of this edge in $D_{0}$ is inverted by some element of $A$. For if $e$ is an edge of $T_{0}$ incident with vertices $u$ and $v$ and if $\alpha \in A$ inverts the projection $e^{G}$ of $e$ in $D_{0}$, then the ordered pair $\left(u^{\alpha}, v^{\alpha}\right)$ is $G$-conjugate to the pair $(v, u)$. Thus, $\alpha i_{g}$ inverts $e$ for some $g \in G$. It follows that $D_{0}^{*}$ can be described in terms of $D_{0}$ (that is, without reference to $T$ ) as the graph obtained by subdividing the edges of $D_{0}$ that are inverted by elements of $A$ (to get an inversion-free $A$-graph) and then forming the quotient graph modulo $A$.

Because $(G(-), D)$ is proper, the $G$-stabilizer of each edge of $T_{0}$ is properly contained in the $G$-stabilizer of both incident vertices unless those vertices are $G$-conjugate. Since $\operatorname{Inn} G \subseteq A$, a similar statement holds for the action of $A$ on $T_{0}$. It remains true for the action of $A$ on $T^{*}$ and so $\left(A(-), D^{*}\right)$ is proper. That $\left(A(-), D^{*}\right)$ also satisfies the EGI hypothesis is immediate from Lemma 3.2.

Statement (ii) of Theorem 1 is implicit in the proof above and the edge number inequality of (iii) follows because the "new" edges produced by subdivision of an $A$-inverted edge are $A$-conjugate. It only remains to verify the second statement in (iii).

Suppose $H$ is a group acting as isometries of a finite directed tree $X$ (with no edge inversions). Arguing by induction on $|V X|$, we claim that the quotient graph $X / H$ is also a tree. For because $X$ is finite, $H$ fixes some vertex $v$ of $X$ and the subtree $\operatorname{star}(v)$ spanned by $\{u \in V X: d(u, v)=1\}$ is then $H$-invariant. If $X_{0}$ is the $H$-tree obtained from $X$ by contracting this subtree, then $X_{0} / H$ is a tree by the inductive hypothesis and thus, $X / H$ is also a tree. This observation applied in the case $X=D$ and $H=A$ implies that $D^{*}$ is a tree if $D$ is a finite tree, thus completing the proof of Theorem 1 .

Theorem 1 applied to the case of a proper HNN extension yields an analog of the Karrass-Pietrowski-Solitar theorem. (See also Theorem 3.3 of [5].) 
Theorem 2 Let $G=H *_{U} t$ be an $H N N$ extension with $H \neq U$ and assume that the subgroup $U$ is conjugate maximal in $H$. Let $A$ be the subgroup of Aut $G$ consisting of all automorphisms which map $H$ to a conjugate of itself. If $B=N_{A u t G}(H) \cap N_{A u t G}\left(H^{t^{-1}}\right)$, then either

(i) $\left|N_{A u t G}(H): B\right|=|H: U|$ and $A$ is an $H N N$ extension $N_{A u t G}(H) *_{B} i_{t}$ (where $i_{t}$ is the inner automorphism of $G$ induced by $t$ ) or

(ii) $\left|N_{\text {Aut } G}(H): B\right|=2|H: U|$ and $A$ is an amalgamated free product $N_{\text {Aut } G}(H) *_{B} D$ where $D=B\langle\delta\rangle$ and $\delta$ interchanges $H$ and $H^{t^{-1}}$ (whence $|D: B|=2)$.

The latter case occurs if and only if $H$ admits an automorphism $\beta$ with $U^{\beta}=U^{t}$ such that the map $t t^{\beta}: U \rightarrow H$ (where $\left.t^{\beta}=\beta^{-1} t \beta\right)$ is the restriction to $U$ of an inner automorphism of $H$.

Proof. Assume that $G$ is an HNN extension $H *_{U} t$ with $H \neq U$ and $U$ conjugate maximal. Here, $D$ is a loop and $A$ is the group of automorphisms of $G$ which map $H$ to a conjugate of itself. Again the hypotheses of Theorem 1 are satisfied. The action of $G$ on $T$ is both vertex and edge-transitive and so, since $A \supseteq \operatorname{Inn} G$, this is certainly true of the action of $A$. Therefore, if the action of $A$ on $T$ is free of inversions and if $p \in V T$ with $H=G_{p}$ then $\left|A_{p}: B\right|=|H: U|$ and $A$ is an HNN-extension $A_{p} *_{B} i_{t}$, where $i_{t}$ is the inner automorphism induced by $t$ and $B=A_{p} \cap\left(A_{p}\right)^{i_{t}^{-1}}$. On the other hand, if there is an inversion then the action of $A$ on the barycentric subdivision $T^{*}$ of $T$ yields the decomposition $A=A_{p} *_{B} A_{r}$, where $r$ is the mid-point of the edge $\left[p, p^{t^{-1}}\right]$. Note that each vertex $p$ of $T$ has $|H: U|$ outgoing edges (all conjugate in $G_{p}$ ) and the same number of incoming edges (again all conjugate in $G_{p}$ ). However, in $T^{*}$ all the (new) edges incident at $p$ have the same orientation relative to $p$ and, because $A$ inverts an edge of $T$, they are all conjugate in $A_{p}$. Thus, in this case $\left|A_{p}: B_{p}\right|=2|H: U|$.

Finally, we claim that the action of $A$ contains an inversion if and only if $H$ admits an automorphism $\beta$ such that $U^{\beta}=U^{t}$ and, for some $a \in H$, $u^{t \beta^{-1} t \beta}=u^{a}$ for all $u \in U$. For with such an automorphism we can define $\alpha \in A$ by setting $h^{\alpha}=\left(h^{\beta^{-1}}\right)^{t}$ for all $h \in H$ and $t^{\alpha}=t^{-1} a^{\beta^{-1}}$. Since $\alpha$ interchanges $H$ and $H^{t}$, it inverts the edge $\left[p, p^{t}\right]$ in $T$. Conversely, if $\alpha$ is an automorphism of $G$ which inverts $\left[p, p^{t}\right]$, then it interchanges $H$ and $H^{t}$, and so if $\gamma=i_{t} \alpha^{-1}$, then $\left.\gamma\right|_{H} \in A u t H$ and $H^{t^{-1} \gamma}=H^{\alpha^{-1}}=H^{t}$. If $\beta=\left.\gamma\right|_{H}$ then, because $U=H \cap H^{t^{-1}}$, it follows that $U^{\beta}=U^{\gamma}=U^{t}$. If $a=t t^{\alpha^{-1}}$, then

$$
H^{a}=H^{t \alpha t \alpha^{-1}}=H
$$

and so $a \in N_{G}(H)=H$. Moreover, for any $u \in U$,

$$
u^{t t^{\beta}}=u^{t \beta^{-1} t \beta}=u^{t \gamma^{-1} t \gamma}=u^{t \alpha t \alpha^{-1}}=u^{a}
$$


and so the proof of Theorem 2 is complete.

In general, while Theorem 1 specifically identifies the vertex groups of $\left(A(-), D^{*}\right)$ as subgroups of $A$, in the absence of further hypotheses about $(G(-), D)$ it seems difficult to obtain structural information about these groups. However, additional assumptions about the $(G(-), D)$ may make such information accessible and yield useful conclusions about the structure of $A$. The remarks in Section 5 provide one illustration of this. In a different vein, if the vertex groups of $(G(-), D)$ in Theorem 1 are all finite then Corollary 5.12 of [3] implies that those of $\left(A(-), D^{*}\right)$ are also finite and so, if $D$ is a finite graph (or more generally, if the orders of the vertex groups are bounded) then $A$ (that is, AutG) is virtually free. (See Theorem IV.1.6 of [2].) D. Varsos has kindly brought to our attention his recent work (with E. Raptis and O. Talelli) on automorphism groups of graph products and, in particular, to [5] which concerns automorphism groups of amalgamated free products and HNN extensions of polycyclic-by-finite groups. Using results from [6] and [3], the proof of the main result of [5] is easily adapted to yield the following generalization:

Theorem 3 Let $G$ and $D$ be as in Theorem 1 and suppose that $D$ is finite. Assume that for any vertex $\mathbf{v}$ of $D$, the vertex group $G(\mathbf{v})$ is polycyclic-byfinite and that each $\mathbf{v}$-incident edge group has finite index in $G(\mathbf{v})$. If $G$ is $\mathbf{Z}$-linear, then Aut $G$ is abelian-by-Z-linear. If $G$ is $\mathbf{Z}$-linear and in addition, in each vertex group the extraction of roots is unique (e.g. if each vertex group is torsion-free nilpotent), then AutG is $\mathbf{Z}$-linear.

Proof. Except for the use of Corollary 5.12 of [3], the proof is identical to that of Corollary 3.5 of [5] which it generalizes. By Theorem 1.3 of [6], the Z-linearity of $G$ implies that the kernel $K$ of the action of $G$ on the standard tree has finite index in each edge group. $G / K$ is, therefore, the fundamental group of a finite graph of finite groups and in particular, is finitely generated and virtually free. But this graph of finite groups satisfies the hypotheses of Corollary 5.12 of [3] and so $\operatorname{Out}(G / K)$ is finite and $\operatorname{Aut}(G / K)$ is finitely generated and virtually free. In particular, $\operatorname{Aut}(G / K)$ is $\mathbf{Z}$-linear. AutK is also Z-linear because $K$ is polycyclic-by-finite. But $K$ is characteristic in $G$ (by Corollary 2.4 of [5]) and so there is a homomorphism from AutG to the Z-linear group $A u t(G / K) \times A u t K$. Since the kernel is abelian (see, for example, Proposition 2.5 of [5]), the first conclusion of the theorem holds. As in the proof of Corollary 3.6 of [5], if the extraction of roots in the vertex groups is unique, then this kernel is trivial and the second conclusion follows.

\section{On a class of HNN extensions}

Let $U$ be a proper subgroup of $H$ and let $G=H *_{U} t$ where we assume that $u^{t}=u$ for all $u \in U$. As before, let $A$ be the group of automorphisms of $G$ 
which map $H$ to a conjugate of itself. Here, using Theorem 2 and a lemma from Section 2, we record a complete description of the structure of $A$ (in terms of $H$ and $U$ ). (It seems very unlikely that these observations are new but we are not aware of a convenient reference, even for the case $U=\{1\}$.)

Since $t$ centralizes $U$, the final statement of Theorem 2 (with $\beta=i d_{H}$ ) implies that $A$ is an amalgamated free product $N_{A u t G}(H) *_{B} D$ where $B=$ $N_{A u t G}(H) \cap N_{A u t G}\left(H^{t^{-1}}\right)$ and $D=B\langle\delta\rangle$ for some $\delta$. In fact, for $\delta$ we may take the automorphism of order 2 defined by $h \mapsto h^{t^{-1}}(\forall h \in H)$ and $t \mapsto t^{-1}$. If $a \in G$, let $i_{a}$ be the inner automorphism of $G$ induced by (conjugation by) $a$ and if $a \in N_{G}(H)$, let $j_{a}=\left.i_{a}\right|_{H} \in A u t H$. If $X \leq G$, let $\operatorname{Inn}_{X}(G)=\left\{i_{x}\right.$ : $x \in X\}$. Also, let $\theta$ be the automorphism of $G$ which fixes elements of $H$ and inverts $t$.

We first analyze the factor $N_{A u t G}(H)$.

If $\alpha$ is any element of $N_{A u t G}(H)$ then by Lemma $2.6, t^{\alpha}=a^{-1} t^{\epsilon} b$ for some $a, b \in H$ and $\epsilon \in\{-1,1\}$ and hence, for some $\beta \in\langle\theta\rangle, t^{\alpha \beta}=a^{-1} t b$. Then

$$
u^{\alpha}=u^{\alpha \beta}=\left(u^{t}\right)^{\alpha \beta}=\left(u^{\alpha \beta}\right)^{t^{\alpha \beta}}=\left(u^{\alpha}\right)^{a^{-1} t b}
$$

and so $\left(u^{\alpha}\right)^{a^{-1}} \in H \cap H^{t^{-1}}=U$ for any $u \in U$. Thus, $\left(U^{\alpha}\right)^{a^{-1}} \subseteq U$. A similar argument using $(\alpha \beta)^{-1}$ instead of $\alpha \beta$ yields that $\left(U^{a}\right)^{\alpha^{-1}} \subseteq U$ and so $\alpha i_{a}^{-1} \in N_{A u t G}(U)$. Moreover, since $t$ centralizes $U,\left(u^{\alpha}\right)^{a^{-1} t b}=\left(u^{\alpha}\right)^{a^{-1} b}$ and so $a^{-1} b \in C_{H}\left(U^{\alpha}\right)=C_{H}\left(U^{a}\right)$, whence $c=a b^{-1} \in C_{H}(U)$.

If $\gamma=\left.\left(\alpha i_{a}^{-1}\right)\right|_{H} \in N_{A u t H}(U)$, let $\phi_{(\gamma, c)}$ be the automorphism of $G$ which maps $h$ to $h^{\gamma}$ for all $h \in H$ and sends $t$ to $t c^{-1}$. Then $\alpha=\phi_{(\gamma, c)} i_{a} \beta=\phi_{(\gamma, c)} \beta i_{a}$. This motivates the following construction:

Let $E_{0}$ be the semidirect product $N_{A u t H}(U)\left[C_{H}(U)\right]$ (using the obvious action). Elements $(\gamma, c)$ of $E_{0}$ are identified with automorphisms $\phi_{(\gamma, c)}$ as above and this defines a homomorphism from $E_{0}$ to $N_{A u t G}(H) \cap N_{A u t G}(U)$. By virtue of this homomorphism, $E_{0}$ acts on $\operatorname{Inn}_{H}(G)$. (In fact, under this action, $C_{H}(U)$ centralizes $\operatorname{Inn}_{H}(G)$ while $N_{A u t H}(U)$ acts in the obvious way.) Thus, we may form a second semidirect product $E_{1}=E_{0}\left[\operatorname{Inn}_{H}(G)\right]$. A simple computation yields that

$$
\theta^{-1}\left(\phi_{(\gamma, c)} i_{a}\right) \theta=\phi_{\left(\gamma j_{c}, c^{-1}\right)} i_{c^{-1} a}
$$

and we may lift this to an action of $\langle\theta\rangle$ on $E_{1}$ (which is trivial on $\operatorname{Inn}_{H}(G)$ ) and construct a corresponding third semidirect product $E=\left[E_{1}\right]\langle\theta\rangle$.

As observed above, the homomorphism $E \rightarrow N_{A u t G}(H)$ defined by

$$
\left(\gamma, c, i_{a}, \beta\right) \mapsto \phi_{(\gamma, c)} i_{a} \beta
$$

is surjective. If $\left(\gamma, c, i_{a}, \beta\right)$ is in the kernel, then $a^{-1} t^{\beta} c^{-1} a=t$. If $\beta=\theta$, then $(t a)^{2}=c^{-1} a^{2} \in H$ which is false. Thus, $\beta=i d_{G}$, whence

$$
a^{t}=c^{-1} a \in H \cap H^{t}=U^{t}
$$

and so $a \in U, \gamma=j_{a}^{-1}$ and $c=1$. We conclude that

$$
N_{A u t G}(U)=E^{\phi} \cong E / K
$$


where $K=\left\{\left(j_{a}^{-1}, 1, i_{a}, i d_{G}\right): a \in U\right\}$.

Next we consider the amalgamated subgroup $B=N_{A}(H) \cap N_{A}\left(H^{t^{-1}}\right)$.

Certainly $B \subseteq N_{A u t G}\left(H \cap H^{t^{-1}}\right)=N_{A u t G}(U)$ and so if $\alpha \in B$ then

$$
t^{\alpha} \in C_{G}\left(U^{\alpha}\right)=C_{G}(U)
$$

. But also, $H^{t^{-1}}=\left(H^{t^{-1}}\right)^{\alpha}=H^{t^{-\alpha}}$ and so

$$
t^{-1} t^{\alpha} \in N_{G}(H) \cap C_{G}(U)=C_{H}(U)
$$

. Therefore, if $\gamma=\left.\alpha\right|_{H} \in N_{A u t H}(U)$ and $t^{-1} t^{\alpha}=c^{-1}$, then

$$
\alpha=\phi_{(\gamma, c)} \in E_{0}
$$

. It follows that $B=\left(E_{0}\right)^{\phi} \cong N_{\text {Aut }(H)}(U)\left[C_{H}(U)\right]$.

It is easily checked that $\delta$ centralizes each $\phi_{(\gamma, 1)}$ and if $c \in C_{H}(U)$, then

$$
\delta^{-1} \phi_{\left(i d_{H}, c\right)} \delta=\phi_{\left(j_{c}, c^{-1}\right)} .
$$

This determines the structure of $D$ as a semidirect product $[B]\langle\theta\rangle$.

Thus, the structure of the factors of $A$ and of the amalgamated subgroup are completely specified in terms of $H$ and $U$.

Because $H \cap Z(G) \subseteq C_{H}(t)=U, H \cap Z(G)=U \cap Z(G)$ and so

$$
\left|\operatorname{Inn}_{H}(G): \operatorname{Inn}_{U}(G)\right|=|H: U|
$$

. Since $K \cong \operatorname{Inn}_{U}(G)$, it follows that if $H$ is finite, then $A$ (which is all of Aut $G$ in this case) is an amalgamated free product of finite groups of order $2\left|N_{\text {Aut } H}(U)\right|\left|C_{H}(U)\right||H: U|$ and $2\left|N_{A u t H}(U)\right|\left|C_{H}(U)\right|$ with the amalgamated subgroup of order $\left|N_{A u t H}(U)\right|\left|C_{H}(U)\right|$.

\section{References}

[1] A. Karrass, A. Pietrowski and D. Solitar, Automorphisms of a free product with amalgamated subgroup, Contemp. Math. 33, Contributions to Group Theory (1984), 328-340.

[2] W. Dicks and M.J. Dunwoody, Groups Acting on Graphs, Cambridge University Press, Cambridge, 1989.

[3] M. Pettet, Virtually free groups with finitely many outer automorphisms, Trans. Amer. Math. Soc., 349 (1997), 4565-4587.

[4] J.-P. Serre, Trees, Springer-Verlag, Berlin, 1980.

[5] E. Raptis and D. Varsos, On the automorphism group of the fundamental group of a graph of polycyclic groups, Algebra Colloquium 4:3 (1997), 241-248.

[6] E. Raptis, O. Talelli and D. Varsos, On finiteness conditions of certain graphs of groups, Int. J. Algebra and Computation 5 (1995), 719-724. 